\title{
On the Unstable Solutions to Functional Vector Differential Equations of the Seventh Order
}

\author{
Cemil Tunç \\ Department of Mathematics, Faculty of Sciences, Yüzüncü Yıl University, 65080 Van, Turkey \\ Correspondence should be addressed to Cemil Tunç; cemtunc@yahoo.com
}

Received 25 August 2012; Accepted 16 September 2012

Academic Editor: Yongsheng S. Han

Copyright (C) 2013 Cemil Tunç. This is an open access article distributed under the Creative Commons Attribution License, which permits unrestricted use, distribution, and reproduction in any medium, provided the original work is properly cited.

This paper studies the instability of the zero solution for a certain nonlinear functional vector differential equation of the seventh order with multiple deviating arguments. Under sufficient conditions, we prove a result on the instability of the zero solution. This work contributes and complements to previously known results in the literature.

\section{Introduction}

More than 100 years ago, the world famous mathematician Lyapunov established the Lyapunov direct method to study stability problems. From then on, the Lyapunov's direct method was also widely used to study the instability of solutions of ordinary differential equations and functional differential equations, see for example, Bereketoğlu [1], Sadek [2], Tejumola [3], Tunç [4-7], C. Tunç and E. Tunç [8], and the references therein. However, a review to date of the literature indicates that the instability of the solutions to the nonlinear functional vector differential equation of the seventh order has not been investigated. This paper is the first work on the subject. It is also known that the expressions of Lyapunov-Krasovskii functional are very complicated and hard to construct. In this paper, we define a LyapunovKrasovskii functional [9] and base on the Krasovskii criteria to prove a new theorem on the topic for the nonlinear functional vector differential equation of the seventh order. In this paper, intend to make a contribution to the subject since the functional differential equations have an important place in various fields of science and engineering.

Meanwhile, some respective contributions on the topic can be summarized as follows.

In 2000, Tejumola [3] discussed the instability of the zero solution of the seventh order scalar nonlinear differential equation without a deviating argument

$$
x^{(7)}+a_{1} x^{(6)}+a_{2} x^{(5)}+a_{3} x^{(4)}
$$

$$
\begin{aligned}
& +\psi_{4}\left(x, x^{\prime}, \ldots, x^{(6)}\right) x^{\prime \prime \prime}+\psi_{5}\left(x^{\prime}\right) x^{\prime \prime} \\
& +\psi_{6}\left(x, x^{\prime}, \ldots, x^{(6)}\right)+\psi_{7}(x)=0 .
\end{aligned}
$$

Later, in 2003, Sadek [2] proved an instability theorem for the seventh order scalar nonlinear differential equation without a deviating argument

$$
\begin{aligned}
& x^{(7)}+a_{1} x^{(6)}+a_{2} x^{(5)}+a_{3} x^{(4)}+a_{4} x^{\prime \prime \prime}+f\left(x^{\prime}\right) x^{\prime \prime} \\
& +g(x) x^{\prime}+h(x)=0 .
\end{aligned}
$$

Recently, Tunç [6, 7] discussed the same problem for the seventh order scalar nonlinear delay differential equations of the form:

$$
\begin{aligned}
x^{(7)} & +a_{1} x^{(6)}+a_{2} x^{(5)}+a_{3} x^{(4)} \\
& +a_{4} x^{\prime \prime \prime}+f\left(x^{\prime}\right) x^{\prime \prime}+g(x) x^{\prime} \\
& +h\left(x, x(t-r), x^{\prime}, \ldots, x^{(6)}(t-r)\right)=0,
\end{aligned}
$$




$$
\begin{aligned}
x^{(7)} & +a_{1} x^{(6)}+a_{2} x^{(5)}+a_{3} x^{(4)} \\
& +\psi_{4}\left(x, x(t-r), x^{\prime}, \ldots, x^{(6)}(t-r)\right) x^{\prime \prime \prime} \\
& +\psi_{5}\left(x^{\prime}\right) x^{\prime \prime}+\psi_{6}\left(x, x(t-r), x^{\prime}, \ldots, x^{(6)}(t-r)\right) \\
& +\psi_{7}(x(t-r))=0 .
\end{aligned}
$$

In this paper, instead of (4), we consider the seventh order nonlinear functional vector differential equation with $n$-deviating arguments, $\tau_{i},(i=1,2, \ldots, n)$ :

$$
\begin{aligned}
X^{(7)} & +A_{1} X^{(6)}+A_{2} X^{(5)}+A_{3} X^{(4)} \\
& +\Psi_{4}\left(X, X\left(t-\tau_{1}\right), \ldots, X\left(t-\tau_{n}\right), \ldots, X^{(6)}\left(t-\tau_{n}\right)\right) \\
& \times X^{\prime \prime \prime}+\Psi_{5}\left(X^{\prime}\right) X^{\prime \prime}+\Psi_{6} \\
& \times\left(X, X\left(t-\tau_{1}\right), \ldots, X\left(t-\tau_{n}\right), \ldots, X^{(6)}\left(t-\tau_{n}\right)\right) X^{\prime} \\
& +\sum_{i=1}^{n} H_{i}\left(X\left(t-\tau_{i}\right)\right)=0 .
\end{aligned}
$$

Setting $X=X_{1}, X^{\prime}=X_{2}, X^{\prime \prime}=X_{3}, X^{\prime \prime \prime}=X_{4}, X^{(4)}=X_{5}$, $X^{(5)}=X_{6}, X^{(6)}=X_{7}$, we can write (5) in system form as follows

$$
\begin{aligned}
X_{1}^{\prime}= & X_{2}, \quad X_{2}^{\prime}=X_{3}, \quad X_{3}^{\prime}=X_{4}, \\
X_{4}^{\prime}= & X_{5}, \quad X_{5}^{\prime}=X_{6}, \quad X_{6}^{\prime}=X_{7}, \\
X_{7}^{\prime}= & -A_{1} X_{7}-A_{2} X_{6}-A_{3} X_{5} \\
& -\Psi_{4}\left(X_{1}, \ldots, X_{1}\left(t-\tau_{n}\right), \ldots, X_{7}\left(t-\tau_{n}\right)\right) X_{4} \\
& -\Psi_{5}\left(X_{2}\right) X_{3} \\
& -\Psi_{6}\left(X_{1}, \ldots, X_{1}\left(t-\tau_{n}\right), \ldots, X_{7}\left(t-\tau_{n}\right)\right) X_{2} \\
& -\sum_{i=1}^{n} H_{i}\left(X_{1}\right)+\sum_{i=1}^{n} \int_{t-\tau_{i}}^{t} J_{H_{i}}\left(X_{1}(s)\right) X_{2}(s) d s,
\end{aligned}
$$

where $\tau_{i}$ are certain positive constants, the fixed delays, $t-\tau_{i}>$ $0, A_{1}, A_{2}$, and $A_{3}$ are constant $n \times n$-symmetric matrices, the primes in (5) denote differentiation with respect to $t, t \in \mathbb{R}_{+}$, $\mathbb{R}_{+}=[0, \infty) ; \Psi_{4}, \Psi_{5}$, and $\Psi_{6}$ are continuous $n \times n$-symmetric matrix functions for the arguments displayed explicitly, $H_{i}$ : $\mathbb{R}^{n} \rightarrow \mathbb{R}^{n}, H_{i}(0)=0, H_{i}\left(X_{1}\right) \neq 0,\left(X_{1} \neq 0\right)$, and $H_{i}$ are continuous for all the respective arguments. The Jacobian matrices of $H_{i}(X)$ are given by

$$
\begin{aligned}
J_{H_{1}}(X) & =\left(\frac{\partial h_{1 i}}{\partial x_{j}}\right), \ldots, J_{H_{n}}(X) \\
& =\left(\frac{\partial h_{n i}}{\partial x_{j}}\right) \quad(i, j=1,2, \ldots, n),
\end{aligned}
$$

where $\left(x_{1}, \ldots, x_{n}\right)$ and $\left(h_{1 i}\right), \ldots,\left(h_{n i}\right)$ are the components of $X$ and $H_{i}$, respectively. It is also assumed that the Jacobian matrices $J_{H_{i}}(X)$ exist and are continuous. The existence and uniqueness of the solutions of (5) are assumed (see Èl'sgol'ts [10, pages 14,15$])$. Throughout what follows that $X_{1}(t), \ldots, X_{7}(t)$ are abbreviated as $X_{1}, \ldots, X_{7}$, respectively.

Let $r \geq 0$ be given, and let $C=C\left([-r, 0], \mathbb{R}^{n}\right)$ with

$$
\|\phi\|=\max _{-r \leq s \leq 0}|\phi(s)|, \quad \phi \in C .
$$

For $H>0$ define $C_{H} \subset C$ by

$$
C_{H}=\{\phi \in C:\|\phi\|<H\} .
$$

If $x:[-r, A) \rightarrow \mathbb{R}^{n}$ is continuous, $0<A \leq \infty$, then, for each $t$ in $[0, A), x_{t}$ in $C$ is defined by

$$
x_{t}(s)=x(t+s), \quad-r \leq s \leq 0, t \geq 0 .
$$

Let $G$ be an open subset of $C$ and consider the general autonomous delay differential system

$$
\dot{x}=F\left(x_{t}\right), \quad x_{t}=x(t+\theta), \quad-r \leq \theta \leq 0, t \geq 0,
$$

where $F: G \rightarrow \mathbb{R}^{n}$ is continuous and maps closed and bounded sets into bounded sets. It follows from the conditions on $F$ that each initial value problem

$$
\dot{x}=F\left(x_{t}\right), \quad x_{0}=\phi \in G
$$

has a unique solution defined on some interval $[0, A), 0<$ $A \leq \infty$. This solution will be denoted by $x(\phi)(\cdot)$ so that $x_{0}(\phi)=\phi$.

Definition 1. The zero solution, $x=0$, of $\dot{x}=F\left(x_{t}\right)$ is stable if for each $\varepsilon>0$ there exists $\delta=\delta(\varepsilon)>0$ such that $\|\phi\|<\delta$ implies that $|x(\phi)(t)|<\varepsilon$ for all $t \geq 0$. The zero solution is said to be unstable if it is not stable.

Consider the linear constant coefficient differential equation of the seventh order:

$$
\begin{aligned}
x^{(7)} & +a_{1} x^{(6)}+a_{2} x^{(5)}+a_{3} x^{(4)}+a_{4} \ddot{x} \\
& +a_{5} \ddot{x}+a_{6} \dot{x}+a_{7} x=0 .
\end{aligned}
$$

It is known from the qualitative behavior of solutions of linear differential equations that the zero solution of (13) is unstable if and only if, the associated auxiliary equation:

$$
\begin{gathered}
\psi(\lambda) \equiv \lambda^{7}+a_{1} \lambda^{6}+a_{2} \lambda^{5}+a_{3} \lambda^{4}+a_{4} \lambda^{3} \\
+a_{5} \lambda^{2}+a_{6} \lambda+a_{7}=0
\end{gathered}
$$

has at least one root with a positive real part. The existence of such a root naturally depends on (though not always all of) the coefficients $a_{1}, a_{2}, \ldots, a_{7}$ in (14). For example, if

$$
a_{1}<0
$$

then it is clear from a consideration of the fact that the sum of the roots of (14) equals $a_{1}$ and that at the least one root of (14) has a positive real part for arbitrary values of $a_{2}, a_{3}, a_{4}$, $a_{5}, a_{6}$, and $a_{7}$. An analogue consideration, combined with the 
fact that the product of the roots (14) equals $\left(-a_{7}\right)$ will verify that at least one root of (14) have a positive real part if

$$
a_{1}=0, \quad a_{7} \neq 0
$$

for arbitrary $a_{2}, a_{3}, a_{4}, a_{5}$ and $a_{6}$. The condition $a_{1}=0$ here in (16) is, however, superfluous when

$$
a_{7}<0
$$

then for $\psi(0)=a_{7}<0$ and $\psi(R)>0$ if $R>0$ is sufficiently large, thus showing that there is a positive real root of (14) subject to (17) and for arbitrary $a_{1}, a_{2}, a_{3}, a_{4}, a_{5}$ and $a_{6}$. Moreover, a necessary and sufficient condition for (14) to has a purely imaginary $\operatorname{root} \lambda=i \beta$ ( $\beta$ real) is that the two equations

$$
\begin{gathered}
-a_{1} \beta^{6}+a_{3} \beta^{4}-a_{5} \beta^{2}+a_{7}=0, \\
-\beta^{6}+a_{2} \beta^{4}-a_{4} \beta^{2}+a_{6}=0
\end{gathered}
$$

are satisfied at the same time. If

$$
a_{1} \leq 0, \quad a_{3} \geq 0, \quad a_{5} \leq 0, \quad a_{7}>0
$$

or

$$
a_{1} \geq 0, \quad a_{3} \leq 0, \quad a_{5} \geq 0, \quad a_{7}<0,
$$

then (14) cannot have any purely imaginary root whatever.

It should be noted that there are no restrictions on the constants $a_{2}, a_{4}$, and $a_{6}$ in (13).

\section{Main Result}

First, we give the following lemma.

Lemma 2. Let $A$ be a real symmetric $n \times n$-matrix and

$$
a^{\prime} \geq \lambda_{i}(A) \geq a>0, \quad(i=1,2, \ldots, n),
$$

where $a$ and $a$ are constants, and $\lambda_{i}(A)$ are the eigenvalues of the matrix A.

Then

$$
\begin{gathered}
a^{\prime}\langle X, X\rangle \geq\langle A X, X\rangle \geq a\langle X, X\rangle, \\
a^{\prime 2}\langle X, X\rangle \geq\langle A X, A X\rangle \geq a^{2}\langle X, X\rangle
\end{gathered}
$$

(Bellman [11]).

Let $\tau=\max \tau_{i}, \quad(i=1,2, \ldots, n)$.

The following theorem is our main result.

Theorem 3. In addition to all the assumptions imposed to $A_{1}$, $A_{2}, A_{3}, \Psi_{4}, \Psi_{5}, \Psi_{6}$, and $H_{i}$ that appear in (5), we assume that there exist constants $a_{2}<0, \alpha_{i}>0, \beta_{i}>0$, and $\delta>0$ such that the conditions

$$
\begin{aligned}
& \lambda_{i}\left(A_{2}\right) \leq a_{2}, \beta_{1} \leq \lambda_{i}\left(J_{H_{1}}\left(X_{1}\right)\right) \\
& \leq \alpha_{1}, \ldots, \beta_{n} \leq \lambda_{i}\left(J_{H_{n}}\left(X_{1}\right)\right) \leq \alpha_{n}, \\
& \frac{1}{4 a_{2}}\left[\lambda_{i}\left(\Psi_{4}(\cdot)\right)\right]^{2}-\lambda_{i}\left(\Psi_{6}(\cdot)\right) \geq \delta>0
\end{aligned}
$$

hold. If $\tau<\delta / \sqrt{n}\left(\alpha_{1}+\cdots+\alpha_{n}\right)$, then the zero solution of (5) is unstable.

Remark 4. For the proof of the theorem, under the conditions sated in the theorem, it suffices to find that there exists a continuous Lyapunov-Krasovskii functional $V(\cdot)=$ $V\left(X_{1 t}, \ldots, X_{7 t}\right)$, which has the Krasovskii properties [9], say $\left(K_{1}\right),\left(K_{2}\right)$, and $\left(K_{3}\right)$.

$\left(K_{1}\right)$ In every neighborhood of $(0,0,0,0,0,0,0)$ there exists a point $\left(\xi_{1}, \ldots, \xi_{7}\right)$ such that $V\left(\xi_{1}, \ldots, \xi_{7}\right)>0$.

$\left(K_{2}\right)$ The time derivative $\dot{V}(\cdot)=(d / d t) V\left(X_{1 t}, \ldots, X_{7 t}\right)$ along solution paths of $(6)$ is positive semidefinite.

$\left(K_{3}\right)$ The only solution $\left(X_{1}, \ldots, X_{7}\right)=\left(X_{1}(t), \ldots, X_{7}(t)\right)$ of (6) which satisfies $(d / d t) V\left(X_{1 t}, \ldots, X_{7 t}\right)=0,(t \geq$ $0)$, is the trivial solution $(0,0,0,0,0,0,0)$.

Proof. Consider the Lyapunov-Krasovskii functional $V(\cdot)=$ $V\left(X_{1 t}, \ldots, X_{7 t}\right)$ defined by

$$
\begin{aligned}
V(\cdot)= & \left\langle X_{7}, X_{2}\right\rangle+\left\langle A_{1} X_{2}, X_{6}\right\rangle \\
& +\left\langle A_{2} X_{2}, X_{5}\right\rangle+\left\langle A_{3} X_{2}, X_{4}\right\rangle \\
& -\left\langle X_{3}, X_{6}\right\rangle-\left\langle A_{1} X_{3}, X_{5}\right\rangle \\
& -\left\langle A_{2} X_{3}, X_{4}\right\rangle+\left\langle X_{4}, X_{5}\right\rangle \\
& -\frac{1}{2}\left\langle A_{3} X_{3}, X_{3}\right\rangle+\frac{1}{2}\left\langle A_{1} X_{4}, X_{4}\right\rangle \\
& +\int_{0}^{1}\left\langle H_{1}\left(\sigma X_{1}\right), X_{1}\right\rangle d \sigma \\
& +\cdots+\int_{0}^{1}\left\langle H_{n}\left(\sigma X_{1}\right), X_{1}\right\rangle d \sigma \\
& +\int_{0}^{1}\left\langle\Psi_{5}\left(\sigma X_{2}\right) X_{2}, X_{2}\right\rangle d \sigma \\
& -\sum_{i=1}^{n} \lambda_{i} \int_{-\tau_{i}}^{0} \int_{t+s}^{t}\left\|X_{2}(\theta)\right\|^{2} d \theta d s,
\end{aligned}
$$

where $s$ is a real variable such that the integrals $\int_{-\tau_{i}}^{0} \int_{t+s}^{t}\left\|X_{2}(\theta)\right\|^{2} d \theta d s$ are nonnegative, and $\lambda_{i}$ are certain positive constants to be determined later in the proof.

We see that

$$
V(0,0,0,0,0,0,0)=0
$$


Since $(\partial / \partial \sigma) H_{i}\left(\sigma X_{1}\right)=J_{H_{i}}\left(\sigma X_{1}\right) X_{1}, H_{i}(0)=0$, then

$$
\begin{aligned}
& \int_{0}^{1}\left\langle H_{1}\left(\sigma X_{1}\right), X_{1}\right\rangle d \sigma \\
& \quad=\iint_{0}^{1}\left\langle\sigma_{1} J_{H_{1}}\left(\sigma_{1} \sigma_{2} X_{1}\right) X_{1}, X_{1}\right\rangle d \sigma_{2} d \sigma_{1} \\
& \quad \geq \beta_{1}\left\|X_{1}\right\|^{2}, \\
& \int_{0}^{1}\left\langle H_{2}\left(\sigma X_{1}\right), X_{1}\right\rangle d \sigma \\
& \quad=\iint_{0}^{1}\left\langle\sigma_{1} J_{H_{2}}\left(\sigma_{1} \sigma_{2} X_{1}\right) X_{1}, X_{1}\right\rangle d \sigma_{2} d \sigma_{1} \\
& \quad \geq \beta_{2}\left\|X_{1}\right\|^{2}, \\
& \quad \vdots \\
& \int_{0}^{1}\left\langle H_{n}\left(\sigma X_{1}\right), X_{1}\right\rangle d \sigma \\
& \quad=\iint_{0}^{1}\left\langle\sigma_{1} J_{H_{n}}\left(\sigma_{1} \sigma_{2} X_{1}\right) X_{1}, X_{1}\right\rangle d \sigma_{2} d \sigma_{1} \\
& \quad \geq \beta_{n}\left\|X_{1}\right\|^{2} .
\end{aligned}
$$

Let

$$
\bar{\varepsilon}=\left(\varepsilon_{11}, \ldots, \varepsilon_{1 n}\right) .
$$

Then, we get

$$
V(\bar{\varepsilon}, 0,0,0,0,0,0)=\left(\sum_{i=1}^{n} \beta_{i}\right)\|\bar{\varepsilon}\|^{2}>0
$$

for all $\bar{\varepsilon}, \bar{\varepsilon} \in \mathbb{R}$, so that every neighborhood of the origin in the $\left(X_{1}, \ldots, X_{7}\right)$-space contains points $\left(\xi_{1}, \ldots, \xi_{7}\right)$ such that $V\left(\xi_{1}, \ldots, \xi_{7}\right)>0$.

Let

$$
\left(X_{1}, \ldots, X_{7}\right)=\left(X_{1}(t), \ldots, X_{7}(t)\right)
$$

be an arbitrary solution of (6).

Calculating the time derivative of the LyapunovKrasovskii functional $V$ along this solution, we obtain

$$
\begin{aligned}
\dot{V}(\cdot)= & \left\langle X_{5}, X_{5}\right\rangle-\left\langle A_{2} X_{4}, X_{4}\right\rangle-\left\langle\Psi_{4}(\cdot) X_{2}, X_{4}\right\rangle \\
& -\left\langle\Psi_{6}(\cdot) X_{2}, X_{2}\right\rangle \\
& +\left\langle X_{2}, \int_{t-\tau_{1}}^{t} J_{H_{1}}\left(X_{1}(s)\right) X_{2}(s) d s\right\rangle \\
& +\cdots+\left\langle X_{2}, \int_{t-\tau_{n}}^{t} J_{H_{n}}\left(X_{1}(s)\right) X_{2}(s) d s\right\rangle \\
& -\left\langle\lambda_{1} \tau_{1} X_{2}, X_{2}\right\rangle-\cdots-\left\langle\lambda_{n} \tau_{n} X_{2}, X_{2}\right\rangle \\
& +\lambda_{1} \int_{t-\tau_{1}}^{t}\left\|X_{2}(\theta)\right\|^{2} d \theta \\
& +\cdots+\lambda_{n} \int_{t-\tau_{n}}^{t}\left\|X_{2}(\theta)\right\|^{2} d \theta .
\end{aligned}
$$

Using the assumption $0<\lambda_{i}\left(J_{H_{i}}\left(X_{1}\right)\right) \leq \alpha_{i}$ and the Schwarz inequality, we have

$$
\begin{aligned}
& \left\langle X_{2}, \int_{t-\tau_{1}}^{t} J_{H_{1}}\left(X_{1}(s)\right) X_{2}(s) d s\right\rangle \\
& \geq-\left\|X_{2}\right\|\left\|\int_{t-\tau_{1}}^{t} J_{H_{1}}\left(X_{1}(s)\right) X_{2}(s) d s\right\| \\
& \geq-\sqrt{n} \alpha_{1}\left\|X_{2}\right\|\left\|\int_{t-\tau_{1}}^{t} X_{2}(s)\right\| d s \\
& \geq-\sqrt{n} \alpha_{1}\left\|X_{2}\right\| \int_{t-\tau_{1}}^{t}\left\|X_{2}(s)\right\| d s \\
& \geq-\frac{1}{2} \sqrt{n} \alpha_{1} \tau_{1}\left\|X_{2}\right\|^{2}-\frac{1}{2} \sqrt{n} \alpha_{1} \int_{t-\tau_{1}}^{t}\left\|X_{2}(s)\right\|^{2} d s, \\
& \left\langle X_{2}, \int_{t-\tau_{2}}^{t} J_{H_{2}}\left(X_{1}(s)\right) X_{2}(s) d s\right\rangle \\
& \geq-\left\|X_{2}\right\|\left\|\int_{t-\tau_{2}}^{t} J_{H_{2}}\left(X_{1}(s)\right) X_{2}(s) d s\right\| \\
& \geq-\sqrt{n} \alpha_{2}\left\|X_{2}\right\|\left\|\int_{t-\tau_{2}}^{t} X_{2}(s)\right\| d s \\
& \geq-\sqrt{n} \alpha_{2}\left\|X_{2}\right\| \int_{t-\tau_{2}}^{t}\left\|X_{2}(s)\right\| d s \\
& \geq-\frac{1}{2} \sqrt{n} \alpha_{2} \tau_{2}\left\|X_{2}\right\|^{2}-\frac{1}{2} \sqrt{n} \alpha_{2} \int_{t-\tau_{2}}^{t}\left\|X_{2}(s)\right\|^{2} d s, \\
& \left\langle X_{2}, \int_{t-\tau_{n}}^{t} J_{H_{n}}\left(X_{1}(s)\right) X_{2}(s) d s\right\rangle \\
& \geq-\left\|X_{2}\right\|\left\|\int_{t-\tau_{n}}^{t} J_{H_{2}}\left(X_{1}(s)\right) X_{2}(s) d s\right\| \\
& \geq-\sqrt{n} \alpha_{n}\left\|X_{2}\right\|\left\|\int_{t-\tau_{n}}^{t} X_{2}(s)\right\| d s \\
& \geq-\sqrt{n} \alpha_{n}\left\|X_{2}\right\| \int_{t-\tau_{n}}^{t}\left\|X_{2}(s)\right\| d s \\
& \geq-\frac{1}{2} \sqrt{n} \alpha_{n} \tau_{n}\left\|X_{2}\right\|^{2}-\frac{1}{2} \sqrt{n} \alpha_{n} \int_{t-\tau_{n}}^{t}\left\|X_{2}(s)\right\|^{2} d s .
\end{aligned}
$$

Hence

$$
\begin{aligned}
\dot{V}(\cdot) \geq & \left\|X_{5}\right\|^{2}-a_{2}\left\|X_{4}+2^{-1} a_{2}^{-1} \Psi_{4}(\cdot) X_{2}\right\|^{2} \\
& +\frac{1}{4 a_{2}}\left\langle\Psi_{4}(\cdot) X_{2}, \Psi_{4}(\cdot) X_{2}\right\rangle-\left\langle\Psi_{6}(\cdot) X_{2}, X_{2}\right\rangle \\
& -\left(\lambda_{1}+\frac{1}{2} \sqrt{n} \alpha_{1}\right) \tau_{1}\left\|X_{2}\right\|^{2}
\end{aligned}
$$




$$
\begin{aligned}
& -\cdots-\left(\lambda_{n}+\frac{1}{2} \sqrt{n} \alpha_{n}\right) \tau_{n}\left\|X_{2}\right\|^{2} \\
& +\left(\lambda_{1}-\frac{1}{2} \sqrt{n} \alpha_{1}\right) \int_{t-\tau_{1}}^{t}\left\|X_{2}(s)\right\|^{2} d s \\
& +\cdots+\left(\lambda_{n}-\frac{1}{2} \sqrt{n} \alpha_{n}\right) \int_{t-\tau_{n}}^{t}\left\|X_{2}(s)\right\|^{2} d s .
\end{aligned}
$$

Let $\lambda_{i}=(1 / 2) \sqrt{n} \alpha_{i}$. Then, using the assumptions of the theorem and the estimate $\tau=\max \tau_{i}$, we have

$$
\begin{aligned}
\dot{V}(\cdot) \geq & \left\|X_{5}\right\|^{2}-a_{2}\left\|X_{4}+2^{-1} a_{2}^{-1} \Psi_{4}(\cdot) X_{2}\right\|^{2} \\
& +\frac{1}{4 a_{2}}\left\langle\Psi_{4}(\cdot) X_{2}, \Psi_{4}(\cdot) X_{2}\right\rangle-\left\langle\Psi_{6}(\cdot) X_{2}, X_{2}\right\rangle \\
& -\sqrt{n} \alpha_{1} \tau_{1}\left\|X_{2}\right\|^{2}-\cdots-\sqrt{n} \alpha_{n} \tau_{n}\left\|X_{2}\right\|^{2} \\
\geq & \left\{\delta-\sqrt{n}\left(\alpha_{1}+\cdots+\alpha_{n}\right) \tau\right\}\left\|X_{2}\right\|^{2} .
\end{aligned}
$$

If $\tau<\delta / \sqrt{n}\left(\alpha_{1}+\cdots+\alpha_{n}\right)$, then, for some positive constant $k$, we have

$$
\dot{V}(\cdot) \geq k\left\|X_{2}\right\|^{2}>0 .
$$

Finally, $\dot{V}(\cdot)=0$ for all $t \geq 0$ necessarily implies that $X_{2}=$ 0 . Hence, it follows that

$$
\begin{gathered}
X_{1}=\xi_{1} \text { (constant vector) }, \quad X_{2}=X^{\prime}=0, \\
X_{3}=X^{\prime \prime}=0, \quad X_{4}=X^{\prime \prime \prime}=0, \quad X_{5}=X^{(4)}=0, \\
X_{6}=X^{(5)}=0, \quad X_{7}=X^{(6)}=0,
\end{gathered}
$$

for all $t \geq 0$ so that

$$
\begin{gathered}
X_{1}=\xi_{1} \text { (constant vector), } \\
X_{2}=X_{3}=X_{4}=X_{5}=X_{6}=X_{7}=0, \quad(t \geq 0) .
\end{gathered}
$$

Therefore, the estimates $(d / d t) V(\cdot)=0$ and (6) imply $X_{1}=$ $X_{2}=X_{3}=X_{4}=X_{5}=X_{6}=X_{7}=0$ since $H_{i}\left(\xi_{1}\right)=0$ if and only if $\xi_{1}=0$. It now follows that functional $V$ thus has all the requisite Krasovskii [9] properties subject to the conditions of the theorem. Hence, we can conclude that the zero solution of (5) is unstable.

The proof of the theorem is completed.

\section{References}

[1] H. Bereketoğlu, "On the periodic solutions of certain class of seventh-order differential equations," Periodica Mathematica Hungarica, vol. 24, no. 1, pp. 13-22, 1992.

[2] A. I. Sadek, "An instability theorem for a certain seventh-order differential equation," Annals of Differential Equations, vol. 19, no. 1, pp. 1-5, 2003.

[3] H. O. Tejumola, "Instability and periodic solutions of certain nonlinear differential equations of orders six and seven," in Proceedings of the National Mathematical Centre on Ordinary Differential Equations, vol. 1, pp. 56-65, Abuja, Nigeria, 2000.
[4] C. Tunç, "A result on the instability of solutions to a class of non-autonomous differential equations of seventh-order," Mathematical Sciences Research Journal, vol. 11, no. 2, pp. 360-366, 2007.

[5] C. Tunç, "New results about instability of nonlinear ordinary vector differential equations of sixth and seventh orders," Dynamics of Continuous, Discrete and Impulsive Systems Series A, vol. 14, no. 1, pp. 123-136, 2007.

[6] C. Tunç, "On the instability of solutions of seventh order nonlinear delay differential equations," Buletinul Academiei de Stiinte a Republicii Moldova, Matematica, vol. 65, no. 1, pp. 60-65, 2011.

[7] C. Tunç, "An instability theorem for a kind of seventh order nonlinear delay differential equations," Annals of Differential Equations, vol. 28, no. 1, pp. 11-14, 2012.

[8] C. Tunç and E. Tunç, "Instability of solutions of certain nonlinear vector differential equations of order seven," Iranian Journal of Science and Technology, Transaction A, vol. 29, no. 3, pp. 515-521, 2005.

[9] N. N. Krasovskii, Stability of Motion: ApplicationS of Lyapunov's Second Method to Differential Systems and equations with Delay, Stanford University Press, Stanford, Calif, USA, 1963, Translated by J. L. Brenner.

[10] L. È. Èl'sgol'ts, Introduction to the Theory of Differential Equations With Deviating Arguments, Holden-Day, San Francisco, Calif, USA, 1966, Translated from the Russian by Robert J. McLaughlin.

[11] R. Bellman, Introduction to Matrix Analysis, vol. 19 of Classics in Applied Mathematics, Society for Industrial and Applied Mathematics, Philadelphia, Pa, USA, 2nd edition, 1997, With a foreword by Gene Golub. 


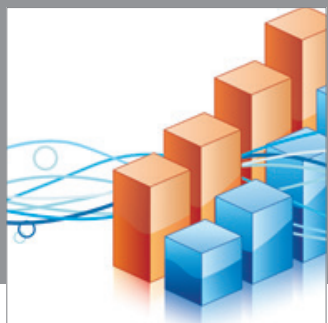

Advances in

Operations Research

mansans

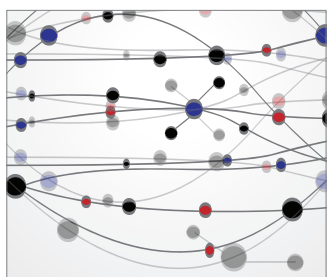

The Scientific World Journal
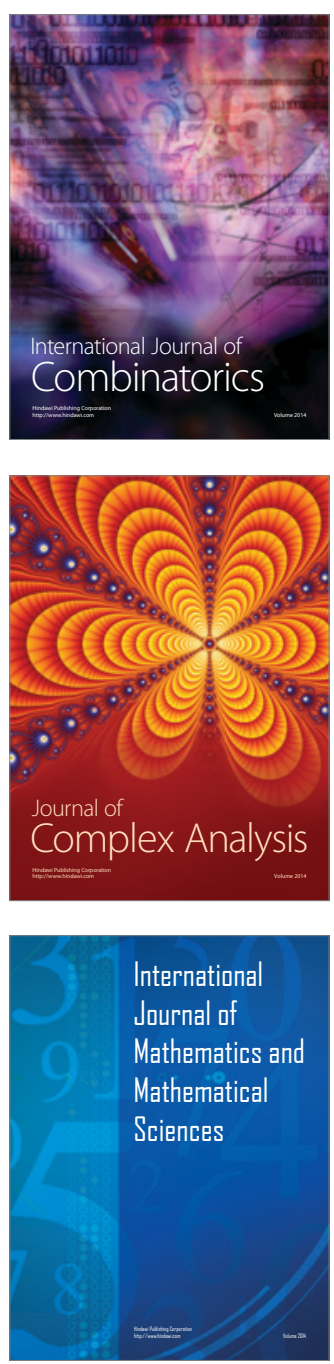
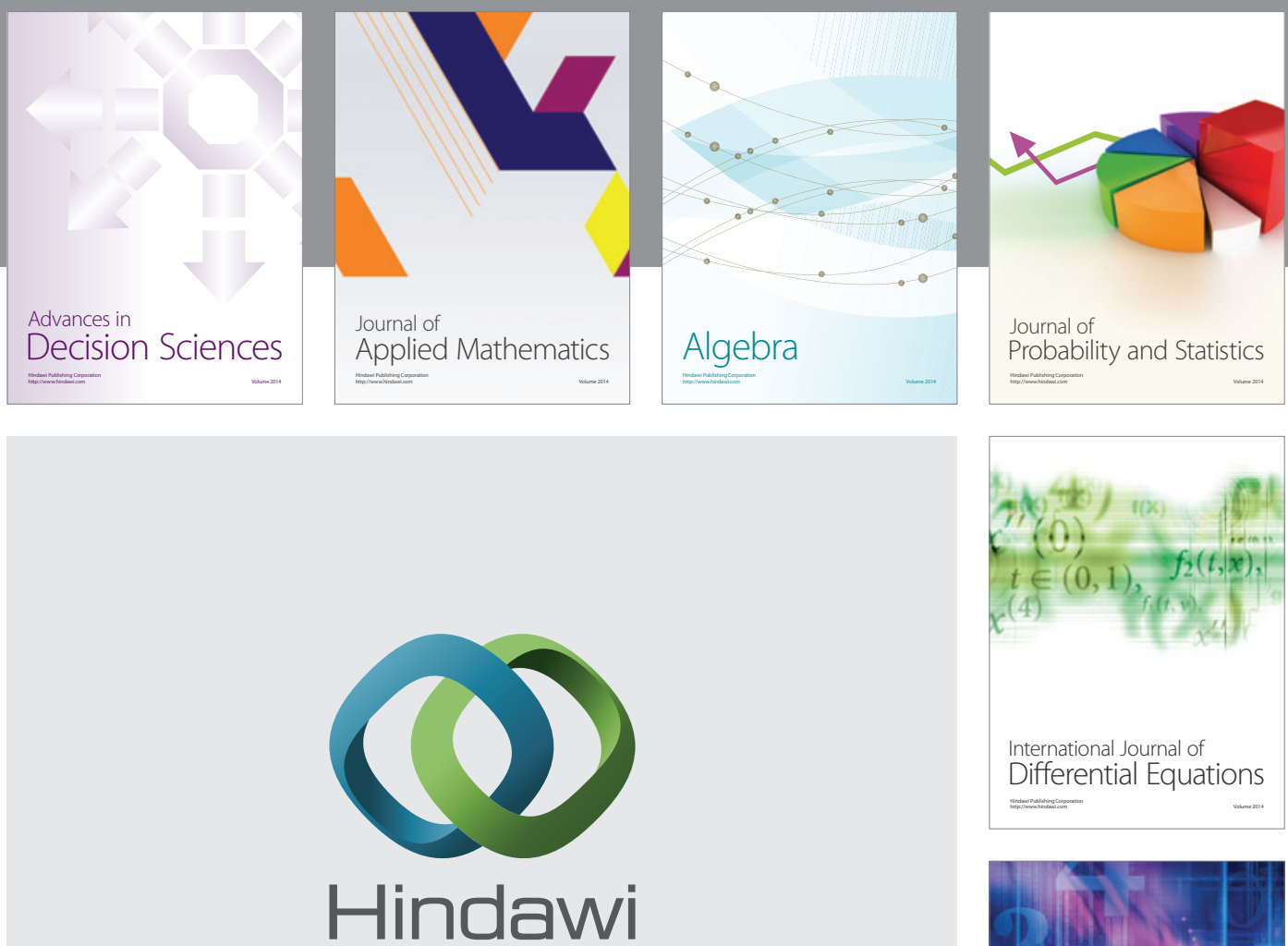

Submit your manuscripts at http://www.hindawi.com
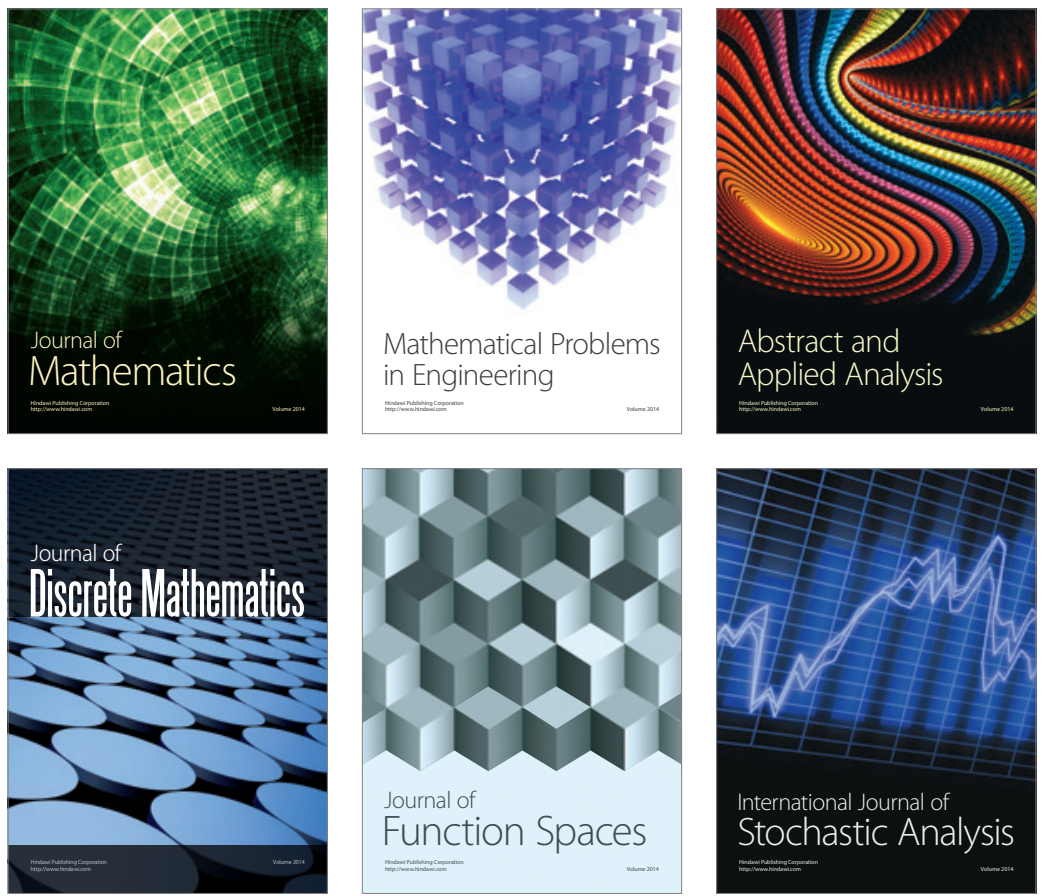

Journal of

Function Spaces

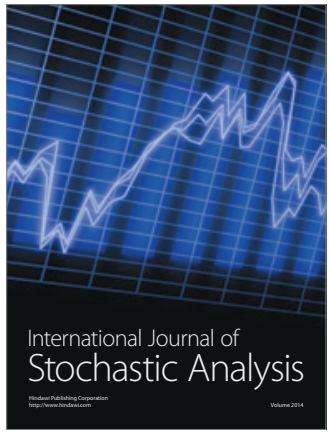

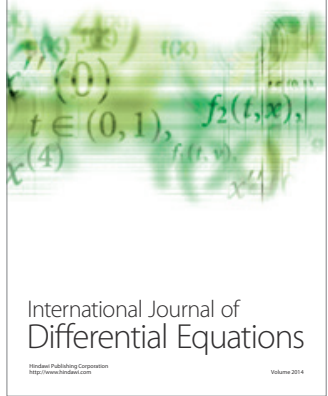
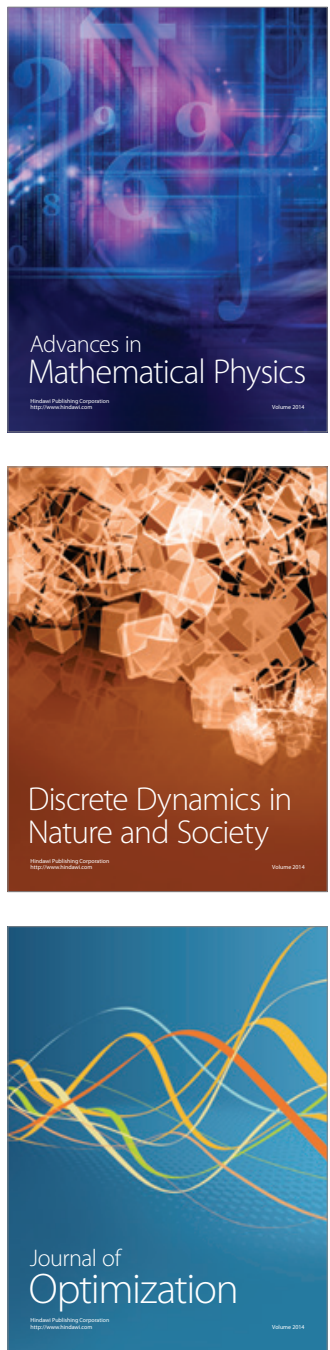